\title{
Successive Relaying Aided Near-Capacity Irregular Distributed Space-Time Coding
}

\author{
Lingkun Kong, Soon Xin Ng, Robert G. Maunder, and Lajos Hanzo \\ School of ECS, University of Southampton, SO17 1BJ, United Kingdom. \\ Tel: +44-23-8059 3125, Fax: +44-23-8059 4508 \\ Email: $\{1 \mathrm{k} 06 \mathrm{r}, \mathrm{sxn}, \mathrm{rm}, \mathrm{hh}\} @$ ecs.soton.ac.uk, http://www-mobile.ecs.soton.ac.uk
}

\begin{abstract}
In this paper, an Irregular Distributed Space-Time (Ir-DST) coding scheme is studied in the context of a twin-relay aided network in which the successive relaying protocol is employed. A tight upperbound of the successive relaying aided network's capacity is given. The distributed codes at the source and relays are jointly designed with the aid of EXtrinsic Information Transfer (EXIT) charts for the sake of high-integrity operation at Signal-to-Noise Ratios (SNRs) close to the corresponding network's capacity. Finally, it is shown that our proposed Ir-DST coding scheme is capable of near-capacity cooperative communications in the successive relaying aided network, which is an explicit benefit of our joint source-and-relay mode design.
\end{abstract}

\section{INTRODUCTION}

\section{A. Background}

Cooperative communications [1], [2] have drawn more and more attentions in the past few years, which combine the benefits of distributed Multiple-Input Multiple-Output (MIMO) systems with relay-aided techniques. In a relay aided network, where the nodes (users) are equipped with either single or multiple antennas, cooperative communications allow the nodes (users) to assist each other in forwarding (relaying) all messages to the destination, rather than transmitting only their own messages. Since the MIMO transmitter's elements in such a network are distributed, the network effectively forms a "distributed MIMO" system. For the sake of improving the diversity gain of relay-aided practical half-duplex-constrained networks, numerous cooperative protocols [1]-[4] have been proposed. However, in most three-terminal cooperative scenarios, a significant multiplexing loss will be incurred compared to direct transmissions due to the half-duplex constraint of practical transceivers. For the sake of recovering the multiplexing loss, a successive relaying protocol was proposed in [5] by incorporating an additional relay in the network and arranging for the two relays to transmit in turn. However, a sophisticated channel code was required for achieving near-error-free detection at the relay nodes and hence to prevent errorpropagation, which was not considered in [5].

Inspired by the classic turbo codes used in non-cooperative communication scenarios, Distributed Turbo Codes (DTC) [6] have been proposed for "distributed MIMO" systems, which benefit from a turbo processing gain. However, DTCs suffer from having an imperfect communication link between the source and relay nodes. Hence a three-component Distributed Turbo Trellis Coded Modulation (DTTCM) scheme has been proposed in [7], which takes into consideration the realistic condition of having an imperfect source-torelay communication link. The DTTCM scheme of [7] was designed using EXtrinsic Information Transfer (EXIT) chart analysis [8], [9], and it was capable of minimizing the decoding error probability at the relay. As a benefit, it performed close to its idealized counterpart that assumes perfect decoding at the relay. However, the DTTCM of [7] still fails to approach the corresponding network's capacity. In [10], an Irregular Distributed Space-Time (Ir-DST) coding scheme was proposed for the sake of approaching the three-terminal relay-aided

The financial support of the China-UK Scholarship, as well as that of the EPSRC UK, the EU under the auspices of the Optimix project is gratefully acknowledged. network's capacity. Due to the half-duplex constraint, the network's effective throughput in [10] was reduced by a factor of two.

\section{B. Contribution}

In this paper, we design an improved Ir-DST coding scheme for a twin-relay aided network, where the successive relaying protocol is employed for the sake of recovering the half-duplex relaying loss. A joint source-and-relay mode design procedure is proposed for the twin-relay aided network. The distributed code components used at the source and relays are optimally designed. Specifically, the Ir-DST coding scheme is capable of approaching the Discreteinput Continuous-output Memoryless Channel's (DCMC) [11], [12] capacity in the context of the successive relaying aided network, while approaching the transmission efficiency of the direct transmission operating without relaying.

The rest of this paper is organised as follows. The system model and the successive relaying protocol are described in Section II. Section III specifies the encoding and decoding processes of the Ir-DST coding scheme designed for the successive relaying aided network. The upper-bound of the network's DCMC capacity and the EXIT chart aided joint source-and-relay mode design are detailed in Section IV, while our simulation results and discussions are provided in Section V. Finally, we conclude in Section VI.

\section{System Model AND Protocol Description}

\section{A. System Model}

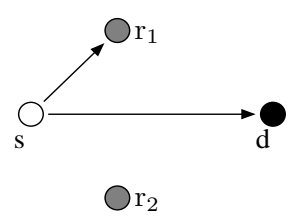

(a) Phase 1

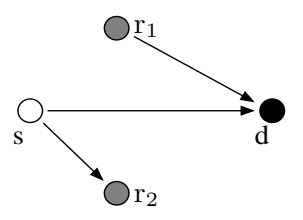

(b) Phase 2

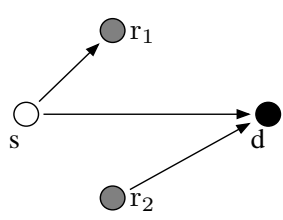

(c) Phase 3
Fig. 1. System model for the successive relaying aided network, where the relays are assumed to be geographically isolated.

We consider the four-terminal successive relaying aided network of Fig. 1, where a single source $s$ is equipped with $N_{s}=2$ antennas, and wants to communicate with the destination $d$ having $N_{d}=4$ antennas. The two relays $r_{1}$ and $r_{2}$ are both equipped with $N_{r_{1}}=N_{r_{2}}=2$ antennas and each relay $r_{i}$ can be either a mobile user or a fixed relay. Compared to the conventional single-relay-aided scheme, it is clearly seen that one more relay is required to support the successive relaying, which potentially increases the overall infrastructure cost. However, this can be avoided when the relays are constituted by inactive mobile users. To obey the practical limitations of practical transceiver design, all nodes in the network obey the half-duplex constraint, i.e. a node cannot transmit and receive simultaneously. Furthermore, we consider a similar scenario to that of [13], where the relays were said to have weak interconnections. In our case, the relays are assumed to be isolated from each other geographically. As in [14], 
we model the communication links between the nodes of Fig. 1 as being subjected to both large-scale free-space path loss as well as to small-scale uncorrelated Rayleigh fading, except for the link between the relays. Hence, as a benefit of the commensurately reduced distance and path loss, we achieve a proportional power-gain [14] for the source-to-relay (SR) links and the relay-to-destination (RD) links with respect to the source-to-destination (SD) link, which are denoted by $G_{s r_{1}}, G_{s r_{2}}$ and $G_{r_{1} d}, G_{r_{2} d}$, respectively. Specifically, the power-gain of the SD link with respect to itself is unity, i.e. $G_{s d}=1$. In this paper, we assume that the relays are closer to the source than to the destination, while both the source and relays are far away from the destination, namely we have $G_{s r_{i}}>G_{r_{i} d}, i=1,2$. In this scenario, the relays benefit from a higher received signal power than the destination, which facilitates the employment of near-perfect Decode-and-Forward (DF) relaying.

\section{B. Protocol Description}

\begin{tabular}{|l|l|l|l|l|}
\hline 1 & 2 & 3 & 4 & 5 \\
\hline
\end{tabular}

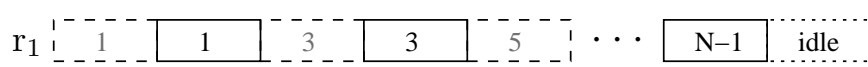

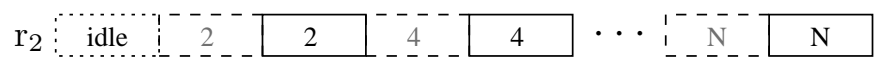

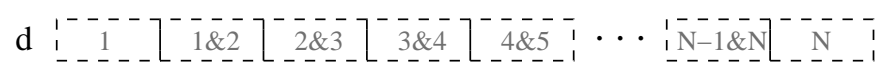

Fig. 2. Frame structure of successive relaying scheme, solid box for transmitted signal, dashed box for received signal and dotted box for idle status.

We split the source transmissions into different identical-length frames. As illustrated in Fig. 1, the transmission scheduling of the successive relaying protocol can be described as follows. In Phase 1 of Fig. 1(a), $s$ transmits frame $1 ; r_{1}$ listens to $s ; r_{2}$ remains silent and $d$ receives frame 1 from $s$. In Phase 2 of Fig. 1(b), $s$ transmits frame $2 ; r_{1}$ decodes, re-encodes and forwards frame $1 ; r_{2}$ listens to $s$ and $d$ receives frame 1 from $r_{1}$ and frame 2 from $s$. By contrast, during Phase 3 seen in Fig. 1(c), $s$ transmits frame 3; $r_{2}$ decodes, re-encodes and forwards frame $2 ; r_{1}$ listens to $s$ and $d$ receives frame 2 from $r_{2}$ and frame 3 from $s$. This progress repeats in this manner until Phase $N$. In Phase $(N+1), s$ and $r_{1}$ (or $r_{2}$ ) keep silent. Then $r_{2}$ (or $r_{1}$ ) decodes, re-encodes and forwards frame $N$ while $d$ receives frame $N$ from $r_{2}$ (or $r_{1}$ ). The frame structure of the successive relaying scheme is further illustrated in Fig. 2. It is clearly seen that, in our successive relaying scheme, $(N+1)$ communication phases are required to convey $N$ frames of information with the aid of two relays. Hence, almost the transmission efficiency of direct transmission is approached, provided that $N$ is sufficiently high. The vector hosting the received signal at the relay $r_{i}$ can be formulated as:

$$
\mathbf{y}_{s r_{i}}=\sqrt{G_{s r_{i}}} \mathbf{H}_{s r_{i}} \mathbf{c}_{s}+\mathbf{n}_{r_{i}}, \quad i=1,2 .
$$

By contrast, the signal vector received at the destination $d$ during the first and last transmission phase can be expressed as:

$$
\mathbf{y}_{s d}=\sqrt{G_{s d}} \mathbf{H}_{s d} \mathbf{c}_{s}+\mathbf{n}_{d}
$$

and

$$
\mathbf{y}_{r_{i} d}=\sqrt{G_{r_{i} d}} \mathbf{H}_{r_{i} d} \mathbf{c}_{r_{i}}+\mathbf{n}_{d}, \quad i=1,2,
$$

respectively, while the signal received during the intermediate phase contains collisions of signals received from the source and relay and is formulated as:

$$
\mathbf{y}_{r_{i} d}=\sqrt{G_{s d}} \mathbf{H}_{s d} \mathbf{c}_{s}+\sqrt{G_{r_{i} d}} \mathbf{H}_{r_{i} d} \mathbf{c}_{r_{i}}+\mathbf{n}_{d}, \quad i=1,2,
$$

where $\mathbf{y}_{s r_{i}}=\left[y_{s r_{i}, 1}, \ldots, y_{s r_{i}, N_{r}}\right]^{T}$ is the $N_{r_{i}}$-element vector of the received signals at the relay $r_{i}$. Furthermore, $\mathbf{y}_{s d}=\left[y_{s d, 1}, \ldots, y_{s d, N_{d}}\right]^{T}$ and $\mathbf{y}_{r_{i} d}=\left[y_{r_{i} d, 1}, \ldots, y_{r_{i} d, N_{d}}\right]^{T}$ are both $N_{d}$-element vectors of the signals received at the destination, while $\mathbf{H}_{s r_{i}} \in \mathbb{C}^{N_{r_{i}} \times N_{s}}, \mathbf{H}_{s d} \in \mathbb{C}^{N_{d} \times N_{s}}$ and $\mathbf{H}_{r_{i} d} \in \mathbb{C}^{N_{d} \times N_{r_{i}}}$ are the corresponding channel matrices having independent and identically complex Gaussian distributed elements with a zero mean and a variance of 0.5 per dimension. Furthermore, $\mathbf{c}_{s}=\left[c_{s, 1}, \ldots, c_{s, N_{s}}\right]^{T}$ and $\mathbf{c}_{r_{i}}=\left[c_{r_{i}, 1}, \ldots, c_{r_{i}, N_{r_{i}}}\right]^{T}$ are the $N_{s}$-element vector of the signals transmitted from the source $s$ and the $N_{r_{i}}$-element vector of the signals transmitted from the relay $r_{i}$, respectively. In this paper, we assume that the source and relays transmit at the same power. Finally, $\mathbf{n}_{r_{i}}=\left[n_{r_{i}, 1}, \ldots, n_{r_{i}, N_{r_{i}}}\right]^{T}$ and $\mathbf{n}_{d}=\left[n_{d, 1}, \ldots, n_{d, N_{d}}\right]^{T}$ are the corresponding $N_{r_{i}}$-element and $N_{d}$-element AWGN vectors, both with each element having a zero mean and a variance of $N_{0} / 2$ per dimension.

\section{IRREGUlar Distributed SPACE-TIME CODING FOR SUCCESSIVE RELAYING NETWORK}

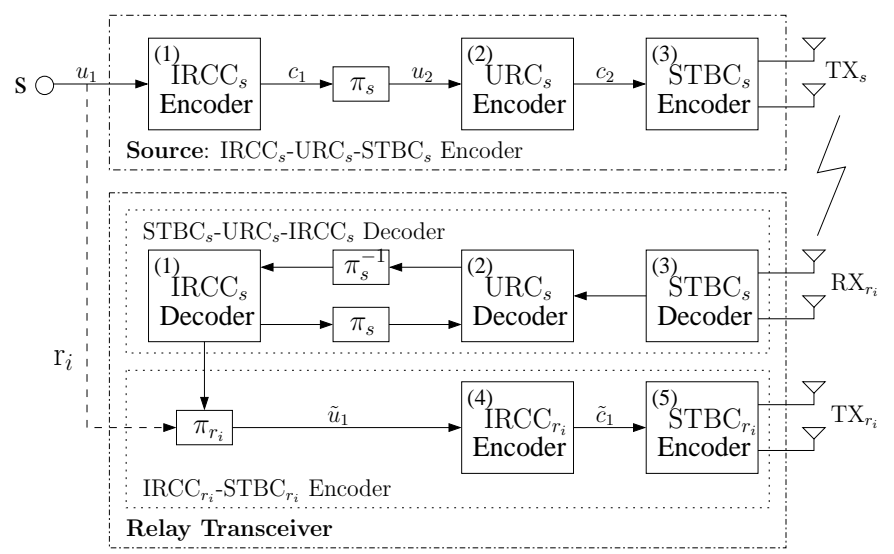

Fig. 3. Schematic of the extended Ir-DST encoder for the successive relaying aided network, where the dashed line indicates the error-free decoding at the relays.

In [10], we have proposed a novel Ir-DST coding scheme for the three-terminal relay-aided network. In this section, we will extend this Ir-DST coding scheme to suit the four-terminal successive relaying aided network.

\section{A. Distributed Encoding at the Source and Relays}

As seen in Fig. 3, at the source of the four-terminal successive relaying aided network, we use the same serial concatenated IRCC-URC-STBC scheme as in [10]. The IRregular Convolutional Code (IRCC) [15], [16] and Unity-Rate Code (URC) [17] are employed to facilitate the near-capacity performance on the end-toend link [18]. On the other hand, the serial concatenated IRCCSTBC scheme at the relay of [10] is employed by both of the two relays in the successive relaying aided network considered in this contribution, where the IRCCs at the relays may have different weighting coefficients and will help to realize the near-networkcapacity performance as in [10]. Since the frames transmitted and relayed from the source and relays have the same frame length as seen in Fig. 2, we choose the same average code rates for the different IRCCs used at the source and relays, namely we have $R_{s}=R_{r_{1}}=R_{r_{2}}$. Note that the relays are assumed to be geographically isolated from each other, as depicted in Fig. 1. Hence, there is no interference between the two relays during any of their transmission phases, as in [13]. Hence, interference suppression is not needed at the relays, as seen in Fig. 3. However, at the destination, except for the first and last phase, suppression of the interference becomes a major problem, which will be detailed in the next subsection. 


\section{B. Iterative Decoding at the Destination}

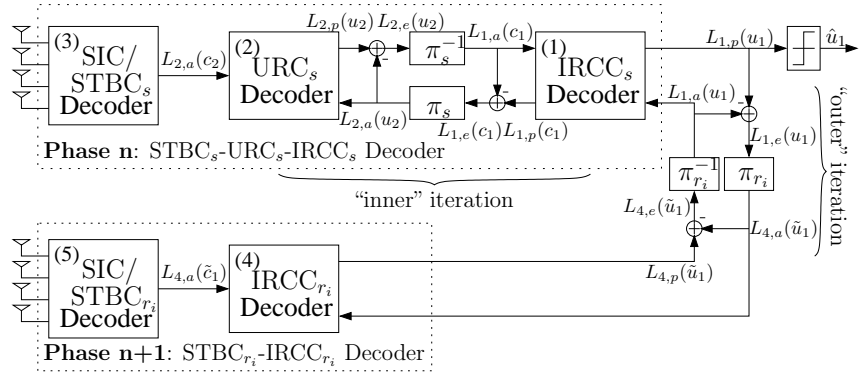

Fig. 4. A frame-by-frame SIC aided iterative decoder of the extended Ir-DST code at the destination.

In contrast to the iterative decoder of the conventional single-relayaided network of [10], Fig. 4 illustrates our novel frame-by-frame successive interference cancellation (SIC) aided iterative decoder designed for the extended Ir-DST coding scheme of the successive relaying aided network. The SIC aided iterative decoder of Fig. 4 has two distinctive parts. Except for the first and last phase of the $(N+1)$ phase relaying protocol, in each intermediate phase, the destination first uses the iterative SIC algorithm [18] to separate the signals received from the source and relays. For example, in Phase $n$ of Fig. 4, the destination receives the signals of frames $(n-1)$ and $n$ from the relay $r_{j}, j=1$ or 2 as well as from the source $s$, respectively. It first detects the signals of frame $(n-1)$ using SIC, while treating the signals of frame $n$ as interference. After frame $(n-1)$ is detected, the destination subtracts it from the received signals and proceeds to detect frame $n$. As discussed in [18], two SIC operations are sufficient to achieve the near-optimum performance for both frames $(n-1)$ and $n$ in this case. After the SIC operations, the detected signals of frame $n$ are passed to the amalgamated "STBC $-\mathrm{URC}_{s}-\mathrm{IRCC}_{s}$ " decoder of Fig. 4, and the separated signals of frame $(n-1)$ are used in the previous three-stage iterative decoding process of Fig. 4 in conjunction with the detected signals of frame $(n-1)$ in Phase $(n-1)$. Similarly, in the subsequent Phase $(n+1)$, the signals of frame $n$ received from the relay $r_{i}, i=2$ or 1 and frame $(n+1)$ received from the source $s$ are detected consecutively by the iterative SIC algorithm at the destination. The detected signals of frame $n$ are passed to the amalgamated "STBC $r_{i}-\mathrm{IRCC}_{r_{i}}$ " decoder of Fig. 4, and the separated signals of frame $(n+1)$ are left to be used in the subsequent three-stage iterative decoding process in conjunction with the detected signals of frame $(n+1)$ in Phase $(n+2)$. The "inner" and "outer" iterations following the SIC operations in Fig. 4 are the same as those presented in [10].

\section{NeAR-CAPACITy System DESIGN BASEd ON EXIT Charts}

As presented in [10], the proposed Ir-DST coding scheme is capable of near-capacity cooperative communications for a conventional single-relay-aided network. In this section, we will demonstrate that our extended Ir-DST coding scheme is also capable of achieving decoding convergence to an infinitesimally low Bit Error Ratio (BER) at the Signal-to-Noise Ratios (SNRs) close to the capacity of the successive relaying aided network. Hence, we first derive the upperbound of the successive relaying aided network's DCMC [11], [12] capacity for Alamouti's STBC scheme in Section IV-A. Then, the EXIT chart based joint source-and-relay mode design will be carried out in Section IV-B. In Section IV-C, the EXIT chart analysis of a specific example will be given to demonstrate the proficiency of the code design procedure proposed in Section IV-B.

\section{A. Upper Bound of Network's Capacity}

We approximate the successive relaying aided network's capacity by the maximum achievable rate attained during the transmission of the source and the independent transmissions of the two relays. Hence, according to the frame structure of Fig. 2, the upper bound of the corresponding network's DCMC [12] capacity can be expressed as follows:

$$
\begin{array}{r}
C_{\mathrm{DCMC}}^{\text {coop }}\left(\mathrm{SNR}_{e}^{\text {coop }}\right)=C_{\mathrm{DCMC}^{s d}}^{\text {con }}\left(\mathrm{SNR}_{e}^{s}+10 \log _{10}\left(G_{s d}\right)\right) \\
+\frac{1}{2} C_{\mathrm{DCMC}_{1} r_{1} d}\left(\mathrm{SNR}_{e}^{r_{1}}+10 \log _{10}\left(G_{r_{1} d}\right)\right) \\
+\frac{1}{2} C_{\mathrm{DCMC}^{r_{2} d}}\left(\operatorname{SNR}_{e}^{r_{2}}+10 \log _{10}\left(G_{r_{2} d}\right)\right)
\end{array}
$$

where we refer to $\mathrm{SNR}_{e}^{s}, \mathrm{SNR}_{e}^{r_{1}}$ and $\mathrm{SNR}_{e}^{r_{2}}$ as the equivalent $\mathrm{SNRs}^{1}$ at the source and relays, respectively, and $\mathrm{SNR}_{e}^{\text {coop }}$ is the equivalent SNR of the successive relaying aided network, where $C_{\mathrm{DCMC}}^{s d}$ and $C_{\mathrm{DCMC}}^{r d}$ are the corresponding SD link's capacity and the RD link's capacity, respectively. They can be computed based on Eq. (9) of [19]. The resultant bandwidth efficiency is determined by normalising the network's capacity upper-bound given by Eq. (5) with respect to the product of the bandwidth $W$ and the signalling period $T$, namely $\eta=C / W T[\mathrm{bit} / \mathrm{s} / \mathrm{Hz}]$, where $W T=1$ for PSK/QAM schemes, when assuming zero Nyquist excess bandwidth. The bandwidth efficiency, $\eta$, is typically plotted against the SNR per bit given by $E_{b} / N_{0}=$ $\mathrm{SNR} / \eta$. For simplicity, we will refer to $\eta$ as the network's capacity.

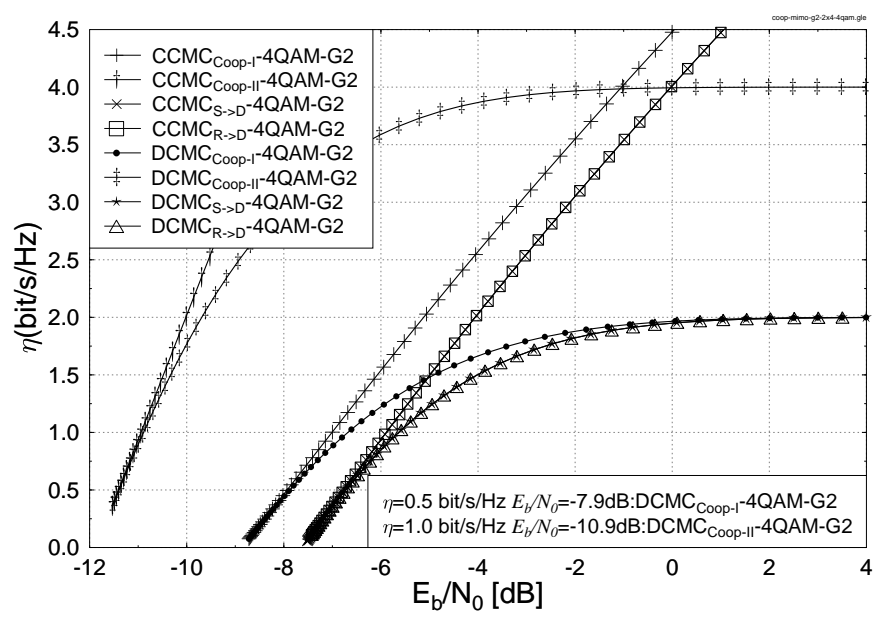

Fig. 5. The capacity curves of different relaying networks employing Alamouti's space-time block code where Coop-I denotes the conventional single-relay-aided network in [10] and Coop-II indicates the successive relaying aided network.

\section{B. Joint Code Design for the Source-and-Relay Nodes}

For the sake of near-capacity cooperative communications in the successive relaying aided network, we extend the joint source-andrelay mode design procedure of [10] to suit our four-terminal network of Fig. 1 in this section, which is summarised as follows:

Step 1: Choose a specific average code rate $R_{S}$ for the $\mathrm{IRCC}_{S}$ at the source and employ the EXIT curve matching algorithm of [15] at the relays to obtain the optimized weighting coefficients $\alpha_{i}, i=1, \ldots, 17$ of $\mathrm{IRCC}_{s}$, where a narrow but marginally open EXIT-tunnel between the EXIT curves of the inner amalgamated " $\mathrm{STBC}_{s}-\mathrm{URC}_{s}$ " decoder of Fig. 4 and the outer $\mathrm{IRCC}_{s}$ decoder emerges at the relays. This implies that a near-capacity performance may be achieved for the SR links. Then store the corresponding required transmit power at the source.

${ }^{1}$ Here we introduced the terminology of equivalent $\mathrm{SNR}_{e}$ to define the ratio of the signal power at the transmitter side with respect to the noise level at the receiver side as in [14]. Although this does not have a direct physical interpretation, it simplifies our discussions. 
Step 2: Choose the same transmit power at the source as stored in Step 1. Fix the optimized $\mathrm{IRCC}_{s}$ weighting coefficients $\alpha_{i}, i=$ $1, \ldots, 17$ obtained in Step 1 at the source. Then perform iterative decoding by exchanging extrinsic information between the amalgamated "STBC ${ }_{s}-\mathrm{URC}_{s}$ " decoder of Fig. 4 and the $\mathrm{IRCC}_{s}$ decoder at the destination, until the further increase of the area $A_{E}$ under the EXIT curve of the amalgamated "STBC ${ }_{s}-\mathrm{URC}_{s}-\mathrm{IRCC}_{s}$ " decoder of Fig. 4 becomes marginal. Then stop this "inner" iterative decoding process.

Step 3: Assume perfectly error-free DF relaying and the same transmit power at the relay $r_{1}$ as that of the source in the second EXIT chart, which examines the evolution of the input/output mutual information exchanges in the three-stage iterative decoder of the IrDST coding scheme. Use the same EXIT curve matching algorithm of [15] to match the SNR-dependent EXIT curve of the amalgamated "STBC $r_{r_{1}}-$ IRCC $_{r_{1}}$ " decoder of Fig. 4 to the target EXIT curve of

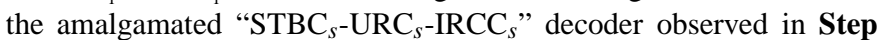
2. If an open EXIT-tunnel fails to appear, increase the transmit power at both the source $s$ and the relay $r_{1}$, until a narrow-but-open EXIT-tunnel emerges. Obtain the optimized weighting coefficients $\beta_{j}, j=1, \ldots, 17$ of $\operatorname{IRCC}_{r_{1}}$.

Step 4: Repeat the operation in Step 3 for the relay $r_{2}$.

Step 5: Finally, choose the higher of the two transmit powers obtained in Step 3 and Step 4 as the ultimate transmit power at the source and relays.

\section{EXIT Charts Analysis}

In this contribution, we consider the same average code rate of 0.5 for the IRCCs at the source and relays, and the specific successive relaying aided network geometry associated with $G_{s r_{1}}=G_{s r_{2}}=8$ and $G_{r_{1} d}=G_{r_{2} d}=2$. Hence, the effective network throughput is $\frac{N}{N+1} R_{S} \log _{2} 4 \approx 1.0 \mathrm{bit} / \mathrm{s} / \mathrm{Hz}$, when 4QAM is employed and the number of frames $N$ is sufficiently high. According to Eq. (5), we derive the corresponding DCMC capacity curve for the successive relaying aided network in Fig. 5, where the single-relay-aided network's capacity curve of [10] is also depicted for comparison. Since the network is geographically symmetrical, we will not differentiate the relays $r_{1}$ and $r_{2}$ for simplicity. Fig. 6 depicts the EXIT chart of the serial concatenated $\mathrm{IRCC}_{s}-\mathrm{URC}_{s}-\mathrm{STBC}_{s}$ scheme of the SR link, where the decoding trajectories are computed based on a frame length of 250000 bits. The EXIT curve of the outer IRCC $_{s}$ having optimized weighting coefficients $\alpha_{i}$ as shown in Fig. 6 was constructed using the curve matching algorithm of [15]. As we can see from Fig. 6, a narrow but marginally open EXIT tunnel emerges for the $(2 \times 2)$ SR communication link. A receive SNR of about $-2.1 \mathrm{~dB}$ is needed in order to attain a decoding convergence to an infinitesimally low BER. Due to the power-gain of the SR communication link, the equivalent SNR at the source can be expressed as:

$$
\mathrm{SNR}_{e}^{s}=\mathrm{SNR}_{r}^{r}-10 \log _{10}\left(G_{s r}\right)[\mathrm{dB}] .
$$

Hence, the minimum $\mathrm{SNR}_{e}^{s}$ at the source required for the sake of obtaining vanishingly low BERs at the relay is $-11.1 \mathrm{~dB}$. Since we assume that the source and relays transmit at the same power, we have $\mathrm{SNR}_{e}^{\text {coop }}=\mathrm{SNR}_{e}^{s}=\mathrm{SNR}_{e}^{r}$. Following the design procedure of Section IV-B, a 'wider-than-necessary' EXIT tunnel is created in the EXIT chart of Fig. 6 at the receive SNR of $-0.7 \mathrm{~dB}$ at the relay, which corresponds to an equivalent SNR of $-9.7 \mathrm{~dB}$ based on Eq. (6) at the source and relays. Accordingly, it is clearly seen in the EXIT chart of Fig. 7 at the destination that after 5 "inner" iterations between the $\operatorname{IRCC}_{S}$ decoder and the amalgamated " $\mathrm{STBC}_{s}-\mathrm{URC}_{s}$ " decoder, the increase of the area $A_{E}$ under the

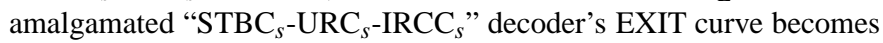
marginal. The resultant matching EXIT curve of the amalgamated

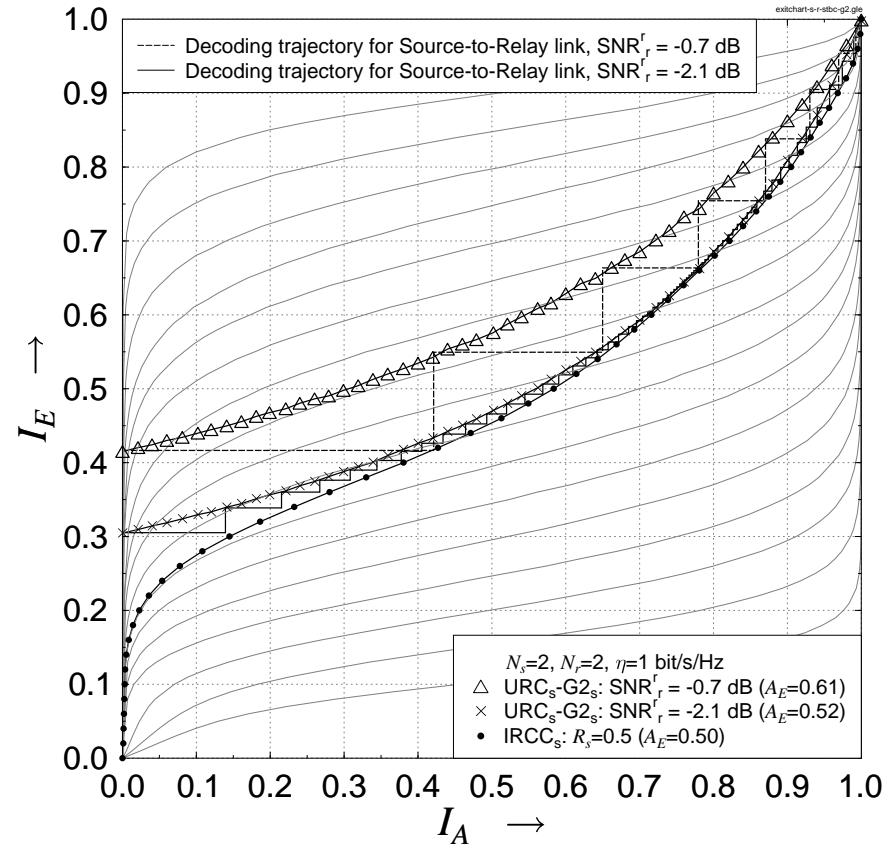

Fig. 6. The EXIT chart curves of the $\mathrm{URC}_{s}-\mathrm{G} 2_{s}$, the $\mathrm{IRCC}_{s}$ with optimized weighting coefficients $\left[\alpha_{1}, \ldots, \alpha_{17}\right]=[0,0,0,0,0,0.327442,0.186505$, $0.113412,0,0.0885527,0,0.0781214,0.0962527,0.0114205,0.0346015$, $0.0136955,0.0500168]$ and 17 IRCC subcodes for the $(2 \times 2)$ SR link where the $\mathrm{SNR}_{r}^{r}$ is the receive $\mathrm{SNR}$ at the relay.

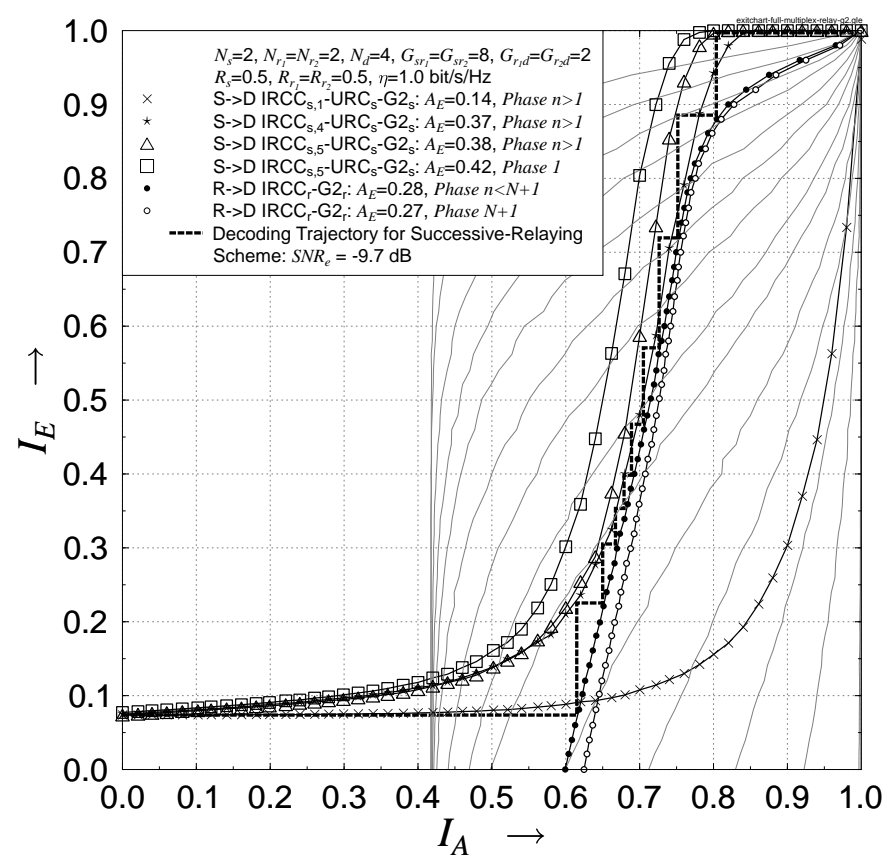

Fig. 7. The EXIT chart curves for the $\mathrm{IRCC}_{s}-\mathrm{URC}_{s}-\mathrm{G} 2_{s}$ with various "inner" iterations, the $\mathrm{IRCC}_{r}-\mathrm{G} 2_{r}$ with $\mathrm{IRCC}_{r}$ having optimized weighting coefficients $\left[\beta_{1}, \ldots, \beta_{17}\right]=[0,0,0,0,0.233115,0.0158742,0.292084,0.220065$, $0.0151108,0,0,0,0,0,0,0,0.22375]$ and 17 SNR-dependent IRCC $_{r}-\mathrm{G}_{r}$ subcodes. The subscript of $\mathrm{IRCC}_{s}$ denotes the number of "inner" iterations between the $\mathrm{IRCC}_{s}$ and " $\mathrm{G} 2_{s}-\mathrm{URC}_{s}$ " decoders and the $\mathrm{SNR}_{e}$ represents the equivalent $\mathrm{SNR}$ at the source and relays. 
"STBC -IRCC $_{r}$ " decoder is shown in Fig. 7, where the $\mathrm{IRCC}_{r}$ has the optimized weighting coefficients $\beta_{j}$ as summarized in Fig. 7. Similarly, as discussed in [10], the 'narrow-but-open' EXIT-tunnel of Fig. 7 indicates the possibility of achieving decoding convergence to an infinitesimally low BER at near-network-capacity SNRs for the Ir-DST coding scheme in the successive relaying aided network. This prediction is verified in Fig. 7 by plotting the corresponding MonteCarlo simulation-based decoding trajectory, which indeed reaches the $(1.0,1.0)$ point of the EXIT chart. Furthermore, we also plot the EXIT curves of the amalgamated "STBC ${ }_{s}-\mathrm{URC}_{s}-\mathrm{IRCC}_{s}$ " decoder in Phase 1 and the amalgamated "STBC $r$-IRCC $r$ " decoder in Phase $(N+1)$ in Fig. 7, respectively. Obviously, since the tunnels in Phase 1 and Phase $(N+1)$ are wider, the trajectories can traverse through the tunnels at a reduced number of iterations to reach the $(1.0,1.0)$ point.

\section{Simulation Results ANd Discussions}

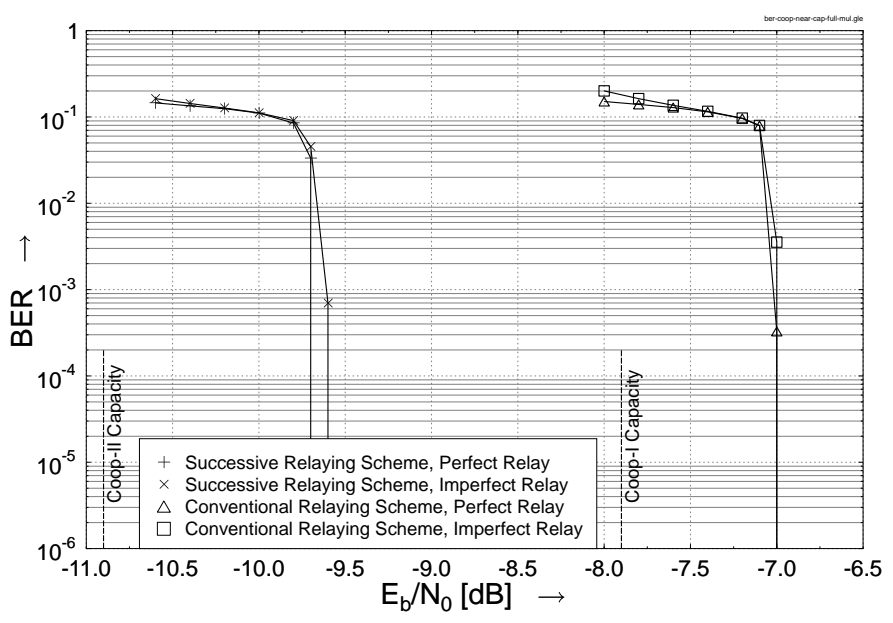

Fig. 8. BER versus $\mathrm{E}_{b} / \mathrm{N}_{0}$ performance of both perfect and imperfect relaying aided Ir-DST coding schemes in the successive relaying aided network for a frame length of 250000 bits, while the performance of the single-relay-aided scheme in [10] is also depicted here for comparison.

In this section, we present the BER versus $\mathrm{E}_{b} / \mathrm{N}_{0}$ performance of both the perfect and imperfect relaying aided Ir-DST coding schemes in the successive relaying aided network in Fig. 8. According to the trajectory predictions seen in Figs. 6 and 7, the number of decoding iterations between the $\mathrm{IRCC}_{s}$ decoder and the amalgamated " $\mathrm{STBC}_{s}$ " $\mathrm{URC}_{s}$ " decoder was fixed to $I^{r}=11$ at the relays. At the destination, the number of "inner" decoding iterations was fixed to $I_{i}^{d}=5$, while the number of "outer" decoding iterations between the parallel amalgamated " $\mathrm{STBC}_{s}-\mathrm{URC}_{s}-\mathrm{IRCC}_{s}$ " decoder and the amalgamated "STBC -IRCC $_{r}$ " decoder was fixed to $I_{o}^{d}=11$. It is clearly seen in Fig. 8 that the performance of the perfect relaying-aided scheme matches the EXIT chart predictions of Fig. 7, while the imperfect relaying-aided scheme performs similarly to the perfect relaying scheme. This is due to the fact that the source information is detected without errors after a sufficiently high number of decoding iterations at the relays. On the other hand, the Ir-DST coding scheme employed in the successive relaying aided network outperforms the single-relayaided scheme by about $2.7 \mathrm{~dB}$ in terms of $E_{b} / N_{0}$. This is because the successive relaying scheme recovers the factor 0.5 multiplexing loss to the same transmission efficiency as direct transmission operating without relaying. As portrayed in Fig. 8, the Ir-DST coding scheme is capable of performing within $-9.7-(-10.9)=1.2 \mathrm{~dB}$ of the corresponding successive relaying aided network's DCMC capacity in terms of $E_{b} / N_{0}$.

\section{CONCLUSIONS}

In this contribution, we have proposed an irregular distributed space-time coding scheme for the successive relaying aided network. The factor 0.5 multiplexing loss in the single-relay-aided network is recovered by the successive relaying protocol with an additional relay. The simulation results show that the joint source-and-relay mode design based on EXIT chart analysis is capable of near-capacity cooperative communications in the twin-relay successive relaying aided network. The scenario where the interference exists between the relays will be considered in our future work. Hence, the interference suppression is needed not only at the destination but also at the relays.

\section{REFERENCES}

[1] A. Sendonaris, E. Erkip, and B. Aazhang, "User cooperation diversity: Part I and II," IEEE Transactions on Communications, vol. 51, pp. 19271948, Nov. 2003.

[2] J. Laneman, D. Tse, and G. Wornell, "Cooperative diversity in wireless networks: Efficient protocols and outage behavior," IEEE Transactions on Information Theory, vol. 50, pp. 3062-3080, Dec. 2004.

[3] J. Laneman and G. Wornell, "Distributed space-time-coded protocols for exploiting cooperative diversity in wireless networks," IEEE Transactions on Information Theory, vol. 49, pp. 2415-2425, Oct. 2003.

[4] K. Azarian, H. El Gamal, and P. Schniter, "On the achievable diversitymultiplexing tradeoff in half-duplex cooperative channels," IEEE Transactions on Information Theory, vol. 51, pp. 4152-4172, Dec. 2005.

[5] Y. Fan, C. Wang, J. Thompson, and H. Poor, "Recovering multiplexing loss through successive relaying using repetition coding," IEEE Transactions on Wireless Communications, vol. 6, pp. 4484-4493, December 2007.

[6] B. Zhao and M. Valenti, "Distributed turbo coded diversity for relay channel," Electronics Letters, vol. 39, pp. 786-787, May 2003.

[7] S. X. Ng, Y. Li and L. Hanzo, "Distributed turbo trellis coded modulation for cooperative communications," in IEEE ICC'09, (Dresden, Germany), 14-18 June 2009.

[8] S. ten Brink, "Convergence behaviour of iteratively decoded parallel concatenated codes," IEEE Transactions on Communications, vol. 49, pp. 1727-1737, Oct. 2001.

[9] S. ten Brink, "Designing iterative decoding schemes with the extrinsic information transfer chart," AEU International Journal of Electronics and Communications, vol. 54, pp. 389-398, Nov. 2000.

[10] L. Kong, S. X. Ng, R. G. Maunder and L. Hanzo, "Irregular Distributed Space-Time Code Design for Near-Capacity Cooperative Communications," in IEEE VTC'09 (Fall), (Anchorage, Alaska, USA), 20-23 September 2009.

[11] J. G. Proakis, Digital Communications. 4th ed. New York: McGraw-Hill, 2001.

[12] S. X. Ng and L. Hanzo, "On the MIMO channel capacity of multidimensional signal sets," IEEE Transactions on Vehicular Technology, vol. 55, pp. 528-536, March 2006.

[13] S. Yang and J.-C. Belfiore, "On slotted amplify-and-forward cooperative diversity schemes," 2006 IEEE International Symposium on Information Theory, pp. 2446-2450, July 2006.

[14] H. Ochiai, P. Mitran, and V. Tarokh, "Design and analysis of collaborative diversity protocols for wireless sensor networks," IEEE 60th Vehicular Technology Conference, 2004 Fall, vol. 7, pp. 4645-4649 Vol. 7, Sept. 2004.

[15] M. Tüchler and J. Hagenauer, "EXIT charts of irregular codes," in Proceeding of the 36th Annual Conference on Information Sciences and Systems [CDROM], (Princeton, NJ, USA), March 2002.

[16] M. Tüchler, "Design of serially concatenated systems depending on the block length," IEEE Transactions on Communications, vol. 52, pp. 209218, Feb. 2004

[17] D. Divsalar, S. Dolinar and F. Pollara, "Serial turbo trellis coded modulation with rate-1 inner code," in ISIT, (Sorrento, Italy), p. 194, 25-30 June 2000.

[18] L. Kong, S. X. Ng and L. Hanzo, "Near-capacity three-stage downlink iteratively decoded generalized layered space-time coding with low complexity," in GLOBECOM'08, (New Orleans, LA, USA), pp. 1-6, 30 Nov.-04 Dec. 2008.

[19] S. X. Ng and S. Das and J. Wang and L. Hanzo, "Near-Capacity Iteratively Decoded Space-Time Block Coding," in IEEE VTC'08 (Spring), (Marina Bay, Singapore), pp. 590-594, 11-14 May 2008. 